\title{
Behandlung bipolarer Erkrankungen
}

n den letzten Jahren hat es eine Reihe von wichtigen therapeutischen Fortschritten in der Behandlung der bipolaren Erkrankung gegeben. Dennoch stellen viele klinische Fragestellungen die behandelnden Ärzte immer noch vor große therapeutische Probleme.

Allen voran ist die Behandlung der bipolaren Depression eine klinische Herausforderung, weil sich Therapiealgorithmen aus der allgemeinen Depressionsbehandlung nicht ohne weiteres auf bipolare Depressionen übertragen lassen. Bei bipolaren Depressionen versagen konventionelle Therapietrategien oft, so dass davon ausgegangen werden muss, dass bis zu 30\% aller Patienten therapierefraktäre Depressionen entwickeln. Ein weiteres Problem ist das Risiko, durch eine antidepressive Medikation ein Umschlagen (switch) in eine Manie auszulösen. Darüber hinaus wurde beobachtet, dass eine antidepressive Behandlung zu einer zunehmenden Verkürzung der Zyklusdauer führen kann und somit Rapid Cycling-Verläufe begünstigt werden. In der Erhaltungstherapie besteht bei bipolaren Depressionen das therapeutische Dilemma zwischen dem Risiko, durch das Fortführen der antidepressiven Medikation eine Manie zu induzieren, und dem Risiko, durch ein rasches Absetzen einen depressiven Rückfall zuzulassen.

In diesem Sonderheft sollen deswegen vorrangig Therapiestrategien bei bipolaren Depressionen vorgestellt werden, mit der die Therapieresponse vergößert werden kann. Dazu zählen die Lithiumaugmentation sowie die Schilddrüsen-Hochdosistherapie, die in jeweils einem Beitrag vorgestellt und diskutiert werden. Psychotherapeutische Maßnahmen spielen neben der Pharmakotherapie eine zunehmend wichtige Rolle in der Behandlung der bipolaren Erkrankung. Ein Beitrag befasst sich deswegen mit psychoedukativen Strategien für diese Patientengruppe.

Fünfzig Prozent aller bipolaren Patienten sind Frauen, und viele trifft die Erkankung im gebärfähigen Alter. Informationen bezüglich sicherer und effizienter Vorgehensweisen sind dringend erforderlich, denn Un- sicherheiten auf ärztlicher Seite können die adäquate Betreuung der Patientinnen erschweren. Spezifische Fragen zu Diagnostik und Therapie bipolarer Depressionen während der Schwangerschaft und Stillzeit sind daher von hohem klinischen Interesse und sollen in einem weiteren Beitrag diskutiert werden.

Schliesslich soll auf eine Neuerung in der Behandlung akuter manischer Episoden eingegangen werden. Atypika werden zunehmend wichtig bei der Therapie von Manien und manisch-depressiven Mischzuständen und scheinen zum Teil eigenständige stimmungsstabilisierende Eigenschaften zu haben. Quetiapin scheint in diesem Zusammenhang eine Substanz darzustellen, deren antimanische Wirkung bei gutem Verträglichkeitsprofil eine sinnvolle Therapiealternative akuter manischer Episoden sein könnte.
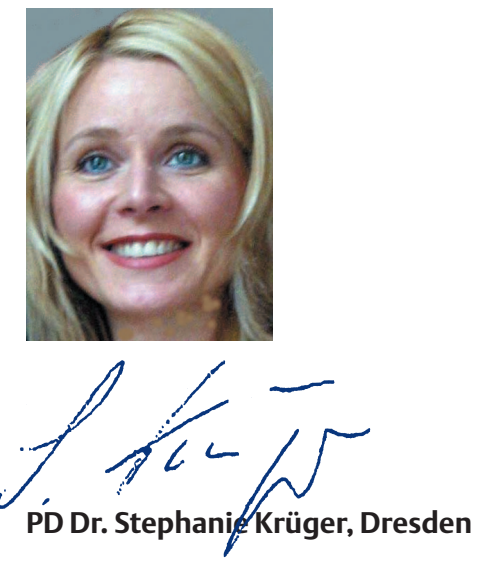